\section{Derechos ambientales y resistencias sociales: El instrumento legal como repertorio contra la minería en Argentina*}

Environmental Rights and social resistances: Legal instrument as a repertoire against mining in Argentina.

\section{LucAs G. Christel **}

\section{Resumen}

En las pasadas dos décadas, la minería a cielo abierto en Argentina se expandió significativamente. Frente a este avance, crecieron fuertemente las resistencias sociales contra la actividad y, desde 2003 a la

\footnotetext{
Este artículo fue posible gracias al apoyo de la Escuela de Política y Gobierno (EPyG - UNSAM) y es parte proyecto PIP CONICET "EN NOMBRE DEL DERECHO: Causas colectivas y movilización legal en la Argentina contemporánea".

** Escuela de Política y Gobierno (EPyG) - Universidad Nacional de San Martín. Avenida 25 de Mayo 1021. Edificio de Ciencias Sociales, Piso $1^{\circ}$ San Martín, Provincia de Buenos Aires, 1650 Argentina. Correo electrónico:lucaschristel@hotmail.com
}

fecha, nueve provincias aprobaron leyes que prohibieron la megaminería.

A partir del estudio de los procesos de sanción de este tipo de normativas en Mendoza y Córdoba, el trabajo analiza la incorporación del instrumento legal al repertorio de acción de las resistencias sociales y el impacto positivo del mismo sobre los procesos de incidencia legislativa.

La incorporación de un discurso legal a las acciones de resistencia, clave para impulsar el avance y reactualización de los derechos ambientales se vio favorecida por tres elementos: la capacidad de los contendientes de complementar acciones contenciosas con otras canalizadas institucionalmente; la pluralidad de actores involucrados y la socialización de experiencias y, por último, por el logro de resultados exitosos.

Palabras clave: Derecho ambiental, resistencias sociales, incidencia legislativa, minería.

\footnotetext{
Abstract

In the past two decades, open pit mining in Argentina expanded significantly. Facing this advance, social resistance against activity grew strongly and, since 2003 to date, nine provinces have passed laws banning mega-mining.

Based on the study of the approval of such laws in Mendoza and Córdoba, this paper analyzes the incorporation of legal instruments as part of the social resistance repertoire and its positive impacts on processes of legislative incidence.
} 
The incorporation of a legal discourse in the actions of resistance-crucial element to promote the advancement and updating of environmental rights- was favored by three elements: the capacity of the disputing parties to complement contentious actions with others institutionally channeled; the plurality of actors involved and the socialization of experiences; and, finally, the achievement of successful results.

Key words: Environmental law, social resistance, legislative incidence, mining.

\section{Introducción}

El desarrollo de la actividad minera a cielo abierto en Argentina ha desencadenado múltiples escenarios de conflicto. En términos sumamente esquemáticos, durante los últimos veinte años, el fuerte crecimiento del sector minero se contrapuso con firmes rechazos sociales a la megaminería'.

La minería fue una de las actividades económicas que más ha crecido durante las últimas dos décadas. A partir de una serie de transformaciones normativas que reconfiguraron el sector a partir de los años 1990, y declarada política de Estado en 2004, la minería en Argentina pasó de representar el $0,22 \%$ del PBI en 1990 hasta alcanzar valores cercanos al 2\% en 2014 (Secretaría de Minería de la Nación, s/d). A la par de este proceso, las

\footnotetext{
La megaminería, contrariamente a la explotación de socavón, requiere la dinamitación de grandes extensiones de montaña y cerros -debido a la baja concentración y al estado de diseminación del mineral- para luego separar la roca del metal mediante la utilización de sustancias químicas como cianuro, ácido sulfúrico o mercurio (Moody, 2007). A lo largo del presente trabajo las expresiones megaminería, minería a cielo abierto son utilizadas como equivalentes.
}

resistencias sociales contra la megaminería se replicaron fuertemente por el territorio argentino y, durante el período comprendido entre los años 2003 y 2011, nueve provincias -poseedoras del dominio originario de los recursos naturalessancionaron leyes que prohibieron el desarrollo de la megaminería a cielo abierto².

Frente a esta situación dual-avance y restricción de la actividad minera, este artículo indaga en la relación entre resistencias sociales, participación política y el avance de los derechos ambientales. Particularmente, se pregunta ¿Qué factores impulsaron la incorporación de un discurso de derecho a las acciones de resistencia?

El presente trabajo parte de tres premisas centrales. En primer lugar, que las resistencias sociales a la minería fueron centrales para la sanción de las leyes de prohibición minera. Este rechazo a la actividad minera en Argentina logró imponer restricciones al avance de una actividad económica considerada estratégica para el gobierno. El accionar conjunto de una pluralidad de actores empeñados en impedir las radicaciones de las empresas mineras impulsó procesos de incidencia exitosa que avanzaron desde las arenas locales hasta alcanzar leyes provinciales de prohibición minera. En segundo lugar, este tipo de normativas de restricción son leyes ambientales claves que ilustran el proceso de avance de la ciudadanía ambiental en Argentina. La mera consagración constitucional

Técnicamente las normativas prohíben el empleo de técnicas esenciales o insumos esenciales para la explotación bajo la modalidad a cielo abierto y no la actividad en sí mismo. A lo largo del trabajo la expresión prohibición o restricción de la minería alude a este tecnicismo. Las leyes fueron: Chubut (abril de 2003); Río Negro (mayo de 2005 - derogada diciembre de 2011); Mendoza (junio de 2007); La Rioja (julio de 2007 - derogada agosto de 2008); Tucumán (julio de 2007); La Pampa (julio de 2007), Córdoba (septiembre de 2008), San Luis (septiembre de 2008) y Tierra del Fuego (abril de 2011). 
de los derechos ambientales dista mucho del efectivo disfrute del derecho a un ambiente sano y, frente a esto, la participación social se vuelve clave en la apropiación y reactualización de los derechos ambientales. En tercer lugar, siendo eje central del trabajo, se afirma que el instrumento legal ha sido incorporado en forma efectiva por las resistencias sociales y es parte fundamental de su repertorio de acción.

A partir del estudio de los casos de Mendoza y Córdoba, se demostrará en este artículo que la incorporación de un discurso de derecho a las acciones de resistencia se vio favorecida por tres elementos: la pluralidad de actores involucrados y la socialización de experiencias; la capacidad de los contendientes de complementar acciones contenciosas ${ }^{3}$ con otras canalizadas institucionalmente; y; por último, los resultados relativamente exitosos conseguidos a partir de esta apelación al elemento legal.

El artículo presenta cuatro apartados centrales. El primer apartado describe en forma breve las particularidades de los derechos ambientales y el rol de la ciudadanía en la interpretación, apropiación y fortalecimiento de los derechos ambientales. Seguidamente, recupera las características centrales de los marcos legales ambientales en Argentina y muestra como los mismos pueden mostrarse incompatibles con un sólido marco normativo minero. El segundo apartado recupera datos e indicadores básicos de la actividad minera Argentina. Seguidamente incorpora también una discusión teórica sobre las resistencias sociales y el proceso progresivo

En términos de Tarrow (2011) lo que hace contenciosa a la acción colectiva es el desafío a otros o a las autoridades de parte de quienes no poseen un acceso regular a las instituciones y cuyos comportamientos de alguna manera resultan disruptivos de las formas "convencionales" de acción política. de incorporación de discursos de derecho a su repertorio de acción. El tercer apartado analiza las experiencias de Mendoza y Córdoba y especifica cómo se ha utilizado el elemento legal por parte de los contendientes durante los conflictos en torno a la minería. Por último, el trabajo cierra con unas conclusiones generales que reflexionan sobre las potencialidades y limitaciones de los derechos ambientales y sus implicancias en la reconfiguración de la acción colectiva ligada a conflictos socioambientales.

El enfoque metodológico del trabajo utiliza el método cualitativo y propone una perspectiva comparativa subnacional (Snyder 2001) a partir de los casos de Mendoza y Córdoba 4 . Con base en técnicas de process-tracing (Collier 2011) son explicadas las principales características de los episodios estudiados, o sus diferencias significativas. El análisis de los casos seleccionados incluyen: más de treinta y cinco entrevista en profundidad, realizadas entre septiembre de 2011 y junio de 2014, con actores de organizaciones sociales, autoridades provinciales y locales e investigadores universitarios; análisis de documentación oficial, de medios nacionales y provinciales; y, finalmente, observación participante.

El caso de Mendoza adquiere su relevancia a partir de dos rasgos principales: en primer lugar, el alto nivel de conflictividad social y la intensidad de las resistencias y, en segunda instancia, un marcado potencial minero, estimado en 350 mil millones de dólares (CAMEM, 2014). Por su parte, Córdoba cuenta con la ley más restrictiva dado que la misma, además de limitar la minería metalífera a cielo abierto, extiende la prohibición a minerales nucleares tales como uranio y torio. 


\section{Derechos Ambientales. Consideraciones teóricas y escenarios legales}

\subsection{Del enunciado constitucional a la práctica concreta.}

Las normas de prohibición de la minería son consideradas leyes ambientales en tanto restringen determinados comportamientos que reconfiguran la relación entre minería y ambiente. Asimismo, y como argumentaremos a continuación, el proceso que desembocó en la sanción de las referidas normativas ilustra el avance de la ciudadanía ambiental en la Argentina.

Una definición genérica de los derechos ambientales los entiende como el derecho humano a gozar de aire, agua y suelo limpios, no solamente para los ciudadanos presentes sino también para las generaciones venideras. Esta concepción de los derechos ambientales como parte de los derechos humanos sostiene que derechos básicos, como la vida y la salud, no pueden ser plenamente disfrutados en entornos ambientales degradados (Shelton 2013). Igualmente, y en estricto orden comparado, los derechos ambientales poseen determinadas características que los distinguen de otros tipos de derechos.

En primer lugar, son derechos de índole colectiva o grupal y no reducibles al goce individual, es decir que no pueden ser sujetos a constricción alguna de tiempo o espacio (Hiskes, 2009). Este carácter colectivo brinda dos implicancias centrales. Por un lado, la responsabilidad intergeneracional, la cual se ve reforzada por el sentido de conexión entre ciudadanos que brindan las garantías constitucionales. Por otro lado, impulsa la posibilidad de reclamar la tutela judicial de los derechos en términos colectivos. Esto implica que no solamente podrán presentar derecho de protección, amparo o tutela de los derechosconstitucionalesquienessonafectados directamente por contaminación ambiental sino también organizaciones o personas físicas que defiendan intereses difusos. De acuerdo a Nogueira Alcalá (2007), los derechos colectivos o difusos emergen de procesos de evolución social y de socialización mediante los cuales emergen grupos de derechos cuyo principal beneficiario es la comunidad en su conjunto, más que una persona en particular. En otras palabras, no hay un titular concreto ni se establecen claramente las prerrogativas para una protección jurídica precisa, de aquí resulta el carácter difuso.

En segundo lugar, los derechos ambientales representan una cuestión de justicia siendo que tanto la contaminación como las políticas ambientales poseen efectos distributivos (Hiskes, 2009). En otras palabras, no todos los ciudadanos, o grupos sociales, se encuentran igualmente expuestos a los riesgos ambientales y, bajo la misma lógica, las políticas ambientales benefician discrecionalmente a determinados sectores.

En tercer lugar, los derechos ambientales incluyen en sí mismo una serie de derechos concomitantes mediante los cuales las organizaciones sociales e individuos pueden clamar por sus legítimos derechos al agua, aire y suelo sano (Hajer 2000, Hiskes 2009). Entre los mismos se encuentran el derecho a obtener información referida a la problemática ambiental, a expresar y difundir información ambiental, a recibir educación sobre derechos ambientales y humanos, a asociarse libremente y pacíficamente en pos de la protección 
ambiental y ser remediado y recompensado ante los daños ambientales (Hiskes, 2009).

La discusión sobre el origen de los derechos constitucionales suele enfatizar la confluencia de factores internos y externos (Gellers 2015) y los procesos de "juridificación" (Magnussen y Banasiak, 2013). Particularmente en la juridificación en el campo ambiental, las expectativas normativas de actores sociales, al entrar dentro del campo del derecho, se transforman en un conjunto de enunciados jurídicos, autorizan o desautorizan ciertas prácticas respecto al ambiente (Azuela, 2006).

Sin embargo, a los efectos de este trabajo resulta de mayor importancia analizar qué sucede una vez garantizado el derecho al ambiente. Siendo que este reconocimiento constitucional no es suficiente para un disfrute efectivo de tal derecho, los principios constitucionales deben ser trasladados a legislaciones habilitantes a los fines de ser efectivamente aplicados (Jeffords y Minkler, 2016; May, 2013). De tal manera, a menos de que a los derechos se les otorgue políticas y prácticas administrativas, serán promesas vacías (Epp 2008) o derechos dormidos (Gargarella 2010).

Ahora bien, en el avance y la pugna por hacer reales los derechos ambientales la clave será la participación. Autores como Hannigan (2006) e Hiskes (2009) enfatizan el rol de los derechos concomitantes y de la participación institucionalizada para explicar el constante proceso de re formulación y el avance de los derechos ambientales. Sin restar importancia a esta mirada, se retomará la premisa sostenida por Christel y Gutiérrez (2017) de que la corporización de los derechos ambientales en prácticas y políticas concretas es impulsada por la combinación de modos contenciosos e institucionalizados de participación política.

La participación ambiental opera como un mecanismo democrático de control social sobre las decisiones políticas y económicas, favorece la inclusión de temas ambientales en la agenda pública $y$, finalmente, hace reales y concretos los derechos ambientales. En esta línea será esperable que los actores sociales se apropien de los derechos ambientales, y de un discurso de derecho, en su afán de búsqueda de normas concretas de protección ambiental en Argentina que enfrenten los intereses mineros.

\subsection{Marcos normativos en tensión: Entre la protección ambiental y la promoción minera.}

A continuación, se hará una breve enumeración de los derechos ambientales, las principales leyes que reglan la actividad minera y, a modo de cierre, la referencia a los principales indicadores que muestran el avance de la actividad minera en Argentina. El país cuenta con sólidos marcos ambientales por un lado y, por otro, un fuerte apoyo a la minería por parte del estado nacional. Esta tendencia contradictoria será clave en el proceso de apropiación de los derechos ambientales por parte de los actores sociales. Como se viera en el apartado anterior, la consagración constitucional del derecho al ambiente sano no es suficiente para su efectivo disfrute. En su afán por restringir la minería, serán las resistencias sociales quienes pondrán en tensión los derechos ambientales con los marcos legales mineros.

Argentina consagra el derecho al ambiente desde 1994, fruto de la última reforma constitucional. El artículo 41 afirma: "el derecho a un ambiente sano, equilibrado, apto para el desarrollo humano 
y para que las actividades productivas satisfagan las necesidades presentes sin comprometer las de las generaciones futuras" Asimismo indica que "corresponde a la Nación dictar las normas que contengan los presupuestos mínimos de protección y a las provincias las necesarias para complementarlas" (Constitución Nacional artículo 41).

Adicionalmente, otra serie de elementos legales consolidan el avance en materia ambiental. En primer lugar, el artículo 43 garantiza la tutela judicial de los derechos. Este recurso, denominado amparo en Argentina - equivalente al recurso de protección garantizado por el Art. 20 de la Constitución de Chile-protege derechos y garantías constitucionales contra todo aquel acto u omisión de autoridades públicas o de particulares que los lesione, restrinja, altere o amenace.

El derecho a una acción de amparo, tutela o protección de derechos, consolida un recurso efectivo, sencillo y rápido para la protección de los derechos fundamentales - incluso en situaciones de emergencia o excepción (Robledo 2010). Estas garantías, a su vez, constituyen una exigencia para todos los Estados que ratificaron de la Convención Americana de Derechos Humanos. De esta manera, estos derechos integran parte esencial del bloque constitucional de derechos en el ámbito latinoamericano (Nogueira Alcalá 2007, Robledo 2010 $)^{5}$

\footnotetext{
"En el contexto latinoamericano, con algunas diferencias en el nomen iuris de la institución la acción de amparo de derechos está presente en los textos constitucionales de Argentina (art. 43); Bolivia (art. 19); Brasil (art 5, LXIX yLXX); Colombia (art. 86); Costa Rica (art. 48); Chile (art. 20); Ecuador (art. 95);El Salvador (art. 247); Guatemala (art. 265); Honduras (art. 183); México (arts.103 y 107); Nicaragua (arts. 45 y 188); Panamá (art. 50); Paraguay (art. 134);Perú (art. 200.2) y Venezuela (art. 27)"(Nogueira Alcalá, 2007:80).
}

Esto permite encuadrar los derechos ambientales como bienes colectivos, habilitando amparos contra cualquier forma de riesgos o vulneración relativa a los derechos ambientales y presentada por el afectado, el defensor del pueblo o las asociaciones que propendan a esos fines.

En segundo lugar, el artículo 124 reconoce el dominio de las provincias sobre los recursos naturales y clarifica la distribución de competencias entre provincias y nación derivada del artículo 41. En ese sentido, la combinación de ambas disposiciones - arts. 41 y 124- induce a pensar que la delegación se efectuó bajo condición de que su ejercicio no importara un vaciamiento del dominio que tienen las provincias sobre esos mismos recursos (Sabsay y Di Paola 2008).

Finalmente, a partir del mandato constitucional de sancionar normas de presupuestos mínimos de protección ambiental, la Ley General de Ambiente $n^{\circ}$ 25.675/02 (LGA), dicta los principios rectores que guían las políticas medioambientales $^{6}$. Se establecen como objetivos de la política ambiental nacional, entre otros: asegurar la preservación, conservación, recuperación y mejoramiento de la calidad de los recursos ambientales; promover el mejoramiento de la calidad de vida de las generaciones presentes y futuras; y prevenir los efectos nocivos o peligrosos que las actividades

\footnotetext{
En base a lo dispuesto por la LGA, en los años subsiguientes a su aprobación se sancionaron normativas de presupuestos mínimos ambientales de gran relevancia, tales como la Ley de Presupuestos Mínimos de Protección Ambiental de los Bosques Nativos $n^{\circ}$ 26.331/07 y Presupuestos Mínimos para la Preservación de los Glaciares y del Ambiente Periglacial $n^{\circ}$ 26.639/10. Para una lectura de la sanción de la Ley de Preservación de Glaciares desde una perspectiva de las soberanías en conflicto ver: Christel y Torunczyk 2017.
} 
antrópicas generan sobre el ambiente posibilitando, además, la sustentabilidad ecológica, económica y social del desarrollo. En su conjunto, estas normas definen el derecho sustantivo al ambiente en Argentina y, como veremos en el desarrollo de los casos, son base fundamental para convertir los enunciados abstractos en prácticas concretas de protección ambiental.

Por su parte, la actividad minera se rige por su código de fondo, es decir el Código de Minería (ley $n^{\circ} 1919$ sancionado en 1886), y es la única actividad económica que cuenta con un código propio. El Código de Minería, de acuerdo a su artículo primero, rige los derechos, obligaciones y procedimientos referentes a la adquisición, explotación y aprovechamiento de las sustancias minerales. No obstante, a los efectos de este análisis resultan más relevantes las reconfiguraciones atravesadas por el sector minero en los años más recientes.

A partir de los primeros años de la década de 1990, el estado argentino llevó adelante un conjunto de reformas que fundaron un nuevo marco jurídico-político. Este proceso de reformas tuvo su correlato en el sector minero con la sanción del Régimen de Inversiones Mineras, ley $n^{\circ} 24.196 / 93$.

En un marco general favorable para el desarrollo de la actividad, dos elementos se destacan en la ley $\mathrm{n}^{\circ} 24.196 / 93$, la estabilidad fiscal y el esquema de regalías. Por un lado, la estabilidad fiscal queda garantizada por 30 años (Artículo 8). En otras palabras, las empresas que desarrollen actividades mineras amparadas bajo esta ley no verán incrementada su carga tributaria total durante tres décadas, a partir de fecha inicio de operaciones.
Por otro lado, el artículo 22 establece que las provincias que perciban, o decidan percibir regalías, no podrán cobrar un porcentaje superior al $3 \%$ sobre el "valor boca mina" del mineral extraído. Posteriormente, la ley $n^{\circ}$ 25.161/99 definió al "valor boca mina" como el valor obtenido en la primera etapa de comercialización, menos los costos de llevar el mineral de "boca mina" a dicha etapa, debiéndose descontar costos de transporte, fletes, seguros, trituración, molienda, fundición, refinación y comercialización. Luego de efectuados estos descuentos, el porcentaje de regalías percibido por las provincias oscila entre el 1,8 y el $2 \%$.

Complementariamente, es preciso retomar el artículo 124 de la Constitución Nacional el cual, como vimos reconoce el dominio originario de las provincias sobre los recursos naturales existentes en sus territorios. Si bien el control de estos recursos siempre estuvo en manos provinciales, el reconocimiento constitucional, a partir de la reforma constitucional de 1994, enfatizó el rol delas provincias como destinatarias del sistema de regalías establecido por el régimen de inversiones mineras. Asimismo, es necesario subrayar que las leyes provinciales de prohibición minera solamente toman sentido a partir de esta potestad subnacional para decidir sobre los usos y explotaciones de sus recursos mineros.

La estructuración de un marco normativo favorable al desarrollo minero tuvo un impacto significativo sobre los principales indicadores del sector. En el año 1990 la producción minera argentina totalizaba 350 millones de dólares, las exportaciones argentinas de minerales no superaban los 35 millones y las inversiones del sector ascendían apenas a 10 millones. La 
actividad como porcentaje del Producto Bruto Interno (PBI), retrocediendo con respecto a años anteriores, se situaba cercano al 0,22\% y, según datos de 1993, la totalidad de las explotaciones mineras llegaban a 130 y los proyectos en curso se encontraban limitados a siete (Cámara de Diputados de la Nación, 1993) ${ }^{7}$. Cerca de dos décadas después, en 2012 la producción nacional minera superó los 6.594 millones de dólares, las exportaciones alcanzaron los 5.412 millones de dólares y las inversiones extranjeras ascendieron a 3.500 millones de la misma moneda (Cámara Argentina de Empresarios Mineros, 2013). Por su parte, para 2011, los emprendimientos mineros en actividad sumaron 614 proyectos en todo concepto (Secretaría de Minería de la Nación, 2012).

Complementado este fortalecimiento del sector minero, se subraya que en materia minera el gobierno de Néstor Kirchner (2003-2007) y los sucesivos de Cristina Fernández de Kirchner (2007-2015) no solamente no modificaron el marco legal heredado de la etapa anterior, sino que profundizaron las tareas de un Estado nacional al servicio de los intereses mineros.

Tres elementos ilustran la apuesta estratégica de los gobiernos kirchneristas por la actividad minera. En primer lugar, el paso del sector minero al influyente Ministerio de Planificación Federal, Inversión Pública y Servicios y su elevación a rango de Secretaría en mayo de 2003. En segundo lugar, y tal vez más importante, la declaración de la minería como política de estado y el lanzamiento del Plan

Anexo presentado por el diputado nacional por San Juan Gómez Centurión -Partido Bloquista- en marco del debate parlamentario de la Ley de Inversiones Mineras 17/3/1993.
Nacional Minero ${ }^{8}$ en enero de 2004. En tercer lugar, el anuncio, en agosto de 2006, del lanzamiento del Plan Nuclear Argentino ${ }^{9}$ con la consiguiente reactivación de la industria nuclear y el incremento de la presión en la búsqueda de uranio en distintos territorios.

\section{Las resistencias sociales a la minería a cielo abierto. Orígenes, características y el elemento jurídico.}

\subsection{Cómo pensar las resistencias sociales.}

Las resistencias sociales han incorporado progresivamente discursos de derecho $y$ estrategias legales a sus repertorios de acción. Esta incorporación fue impulsada por tres factores, que combinando y reforzándose entre sí, favorecieron dicho proceso: la pluralidad de actores involucrados y la socialización de experiencias; la capacidad de los contendientes de complementar acciones contenciosas con otras canalizadas institucionalmente; y; por último, los resultados relativamente exitosos conseguidos a partir de esta apelación al elemento legal.

Primero, es necesario abordar ciertas cuestiones conceptuales de las resistencias sociales, reparando en sus particularidades y características distintivas. Seguidamente

\footnotetext{
Los lineamientos centrales del Plan Nacional Minero son: minería como política de estado; escenarios previsibles para la inversión; impulso al modelo productivo nacional; relación producción y comunidad; integración regional; democratización de la información pública (Secretaría de Minería s/d ).

9 La propuesta de reactivación de la actividad atómica se basó sobre cuatro pilares básicos: la finalización de la central Atucha II; la posibilidad de construcción de una cuarta generadora nuclear; la extensión de la vida útil de la central de Embalse; y la reanudación de la producción de uranio enriquecido (CNEA 2011).
} 
veremos cómo se relacionan las mismas con los tres factores que impulsaron la incorporación del elemento legal a los repertorios de acción de las resistencias.

Los actores más movilizados y comprometidos en la lucha contra la megaminería en Argentina han sido las asambleas ciudadanas y/o socioambientales. Mayoritariamente, los distintos trabajos sobre la temática han hecho foco en estos grupos, destacando su carácter asambleario, horizontal, apartidario, así como también su preferencia por una actuación por fuera de los canales institucionales (Svampa y Antonelli 2009; Svampa et al. 2009, Delamata 2009, entre otros), conceptualizándolas como un nuevo ambientalismo de base (Gutiérrez 2016).

Destacando el rol clave jugado por las asambleas socioambientales durante los conflictos, así como también los aportes teóricos y empíricos de los estudios señalados, se afirma que el estudio de las resistencias no puede centrarse únicamente en el actor asambleario. Por lo tanto, las resistencias sociales serán entendidas como conjunto de acciones directas y acciones mediadas por canales institucionales, tendientes a un objetivo específico y llevadas a cabo en un espacio delimitado, que una pluralidad de actores desarrolla en oposición a intereses estatales y/o privados (Christel 2016:6).

Definir las resistencias sociales en términos de acciones acerca a dos de los factores que impulsaron la incorporación del instrumento legal como parte del repertorio de las mismas. Por un lado, la pluralidad de actores involucrados y, por otro lado, la capacidad de combinar acciones directas con acciones institucionalizadas.
En primer lugar, focalizar sobre las acciones de resistencia permite incluir al análisis, además del accionar de los grupos más comprometidos, actuaciones intermitentes, esporádicas o menores de otros actores involucrados en las resistencias. Así, es posible dar cuenta de la incorporación a las acciones de resistencia de múltiples y variados actores, tales como organizaciones no gubernamentales, actores económicos, abogados ambientalistas ${ }^{10}$ e incluso funcionarios estatales. Algunos de estos actores son más cercanos al conocimiento legal, máxime los abogados expertos en materia ambiental. La confluencia, esporádica o más permanente según el caso, de actores disímiles facilitó la socialización de saberes, conocimientos y experiencias e impulsó la difusión de elementos legales y un discurso de derecho.

En segundo lugar, las resistencias sociales no agotan su repertorio en la acción directa, sino que la complementan con una acción institucional derivada de su vinculación con los actores estatales. Si bien el grueso de las acciones encabezadas por los actores más movilizados, las asambleas ambientales, fueron de tinte contencioso, la canalización de acciones institucionales ha sido importante en los procesos de resistencia y rechazo a la actividad minera. Esto se ha dado principalmente en dos direcciones. Por un lado, dentro de la pluralidad amplia de actores, ciertos grupos como ONGs o actores económicos, tradicionalmente se han sentido más cercanos

\footnotetext{
Los abogados ambientalistas pueden ser conceptualizados como organizaciones de apoyo (Kriesi 1996), dado que aportan recursos claves para los actores movilizados pero sin necesariamente involucrarse en la acción directa. En el caso de las resistencias a la minería se destaca la actuación de la Asociación Argentina de Abogados Ambientalistas (AAdeAA) y la Red de Asistencia Juridica contra la Megamería (REDAJ).
} 
a la participación institucional. Por otro lado, los actores de base u organizaciones asamblearias, durante el transcurso de los conflictos han aprendido a complementar sus prácticas, usualmente contenciosas, con herramientas institucionales de participación, sin que una modalidad implique el debilitamiento de la otra. Esta combinación entre acción contenciosa y participación institucional va en línea con lo señalado por Christel y Gutiérrez (2017) respecto de los factores que impulsan el avance de los derechos ambientales.

Adicionalmente, se realizan dos aclaraciones respecto de la especificidad de las resistencias sociales. Siendo que estas últimas buscan limitar y/o prohibir la actividad minera a cielo abierto, la noción de especificidad del objetivo común y espacio geográficamente delimitado facilitan el estudio de los sucesos bajo estudio. En otras palabras, existe un mayor grado de especificidad en pretender la prohibición de la megaminería en Mendoza, que al perseguir el resguardo ecológico o la modificación de las relaciones entre el hombre y la naturaleza, aplicable por ejemplo al movimiento ambiental. Por su parte, el espacio delimitado caracteriza un ámbito de acción de las resistencias acotado geográficamente. La mayoría de las acciones de resistencia tienen lugar en un espacio físico delimitado por las fronteras provinciales y su eventual éxito, medido en función de la sanción de leyes de restricción minera, es igualmente restringido a dicha la arena subnacional.

\subsection{El surgimiento de las resistencias sociales}

A continuación, y a partir de una breve descripción del surgimiento de las primeras expresiones de rechazo a la actividad minera en Argentina, se refleja el restante elemento que favoreció la incorporación del elemento legal al repertorio de las resistencias sociales: los logros conseguidos.

Los sucesos de Esquel, Chubut, constituyen un punto de inicio para el análisis de la protesta social contra la megaminería en Argentina (Svampa y Antonelli, 2009; Marín, 2009; Schein, 2015). En noviembre de 2002 más de 600 vecinos conformaron la Asamblea de Vecinos Autoconvocados (AVA) por el "No a la Mina" ante la intención de Meridian Gold de instalar una mina de oro a $20 \mathrm{~km}$ del casco urbano.

En un clima de tensión y preocupación creciente, el 16 de diciembre de 2002 "No a la Mina" inauguró la estrategia legal con la presentación de un amparo ambiental solicitando el cese del emprendimiento. Tal presentación tuvo como desenlace una medida cautelar y luego, en febrero de 2003, el juez civil paralizó el proyecto mediante sentencia definitiva ${ }^{11}$. A la par de esta estrategia legal, un estado de movilización masivo y casi permanente forzó la convocatoria a un referéndum municipal sobre el emprendimiento minero.

El 23 de marzo de 2003, la población de Esquel se expresó en el referéndum sobre la explotación o no de la actividad minera y el resultado fue concluyente: un $81 \%$ se decidió por el "No a la mina". Este proceso inauguró un proceso de incidencia exitosa sobre la legislación provincial. El 9 de abril del mencionado año Chubut sancionó la ley $n^{\circ} 5.001$ mediante la

\footnotetext{
Posteriormente el fallo sería confirmado por la Cámara de Apelaciones local, el Supremo Tribunal de Justicia de Chubut y, finalmente, en abril de 2007 por la Corte Suprema de Justicia de la Nación). El argumento esgrimido en el fallo original y en las subsiguientes confirmaciones se centró en la aplicación directa del principio precautorio ambiental.
} 
cual se prohibió la explotación minera a cielo abierto y con utilización de cianuro ${ }^{12}$.

Luego de los sucesos de Esquel, ante los proyectos de nuevos emprendimientos mineros en otras provincias se comenzaron a organizar resistencias. Agrupadas inicialmente en pequeñas organizaciones autoconvocadas, se articularon luego regionalmente hasta constituir colectivos nacionales en defensa del ambiente. Un caso ilustrativo de estas construcciones es la Unión de Asambleas Ciudadanas ${ }^{13}$ (UAC), surgida en Córdoba en Julio de 2006. Este espacio, integrado por diversas asambleas, grupos de autoconvocadosy colectivos sociales, también ha potenciado el proceso de compartir experiencias de resistencia y socialización de conocimientos técnicos y legales (Unión de Asambleas Ciudadanas, 2011).

Luego del gran logro de la movilización social de Esquel y su incidencia sobre la legislación de Chubut, poco más de dos años pasaron para que se sancionase en Argentina una normativa similar. En julio de 2005, la legislatura de Río Negro, en un contexto de creciente preocupación social y expresiones ciudadanas de resistencia, aprobó la ley $n^{\circ} 3.981$ de restricción de la explotación minera a cielo abierto con métodos químicos.

12 La ley llamó a realizar una zonificación del territorio provincial, abriendo la posibilidad de permitir la explotación de minería a cielo abierto en determinadas zonas. El gobierno de Chubut presentó recién en diciembre de 2011 el primer informe preliminar con las zonas habilitadas para la explotación minera a cielo abierto y el conflicto social continúa latente.

13 "La Unión de Asambleas Ciudadanas es un espacio de encuentro y organización de asambleas socio-ambientales y organizaciones autónomas que fueron creciendo a lo largo y a lo ancho de todo el país, que enfrentan a las transnacionales y las políticas de recolonización que saquean y destruyen nuestros bienes comunes, nuestras culturas y nuestros pueblos" (Unión de Asambleas Ciudadanas, 2011).
El punto más álgido de la relación entre resistencias sociales y sanción de leyes de restricción minera transcurrió entre los años 2007 y 2008. En un contexto de aumento de la conflictividad social, durante esos años, las provincias de La Rioja, Tucumán, Mendoza, La Pampa, Córdoba y San Luis sancionaron normas que prohibieron la megaminería a cielo abierto.

\section{Las experiencias de Mendoza y Córdoba. Repertorios jurídicos e incidencia legislativa.}

Mendoza y Córdoba son dos de las siete provincias argentinas que actualmente prohíben en sus territorios la explotación minera metalífera a cielo abierto. En ambos casos las resistencias sociales han incidido positivamente sobre las legislaciones. Mientras Mendoza sancionó la ley 7.722 en junio de 2007, Córdoba hizo lo propio en septiembre de 2008, prohibiendo incluso la explotación de toda minería nuclear. A continuación, se reconstruyen las experiencias de ambas provincias, enfatizando en la incorporación del elemento legal por parte de los actores sociales y la importancia del mismo durante el trascurso del conflicto socioambiental.

\subsection{El caso de Mendoza}

La experiencia mendocina se caracteriza, en términos esquemático por tres elementos centrales: La existencia de fuertes focos de rechazo a la minería en San Carlos y el Valle de $\mathrm{Uco}^{14}$, San Rafael y General Alvear; una importante presencia de actores económicos

El Valle de Uco, donde se ubican estas localidades, constituye uno de los tres grandes oasis urbanos y agrícolas del mapa provincial: el oasis norte (Gran Mendoza y Zona Este), el oasis de Valle de Uco (centro) y el oasis Sur (San Rafael y General Alvear). 
en las acciones de resistencia, principalmente pequeños y medianos productores agrícolas; $y$, por último, la existencia de avances y retrocesos en términos de limitación normativa de la minería, incluyendo la sanción de ordenanzas municipales exitosas y la derogación de una ley provincial de prohibición minera mediante un decreto del ejecutivo.

Las primeras alertas por el tema minero en Mendoza tuvieron lugar en 2003 en la ciudad de San Carlos cuando se advirtió la presencia de mineras operando en los cerros de la zona (Wagner 2010). Luego, en 2005, creció la conflictividad al conocerse las intenciones de la empresa Tenke de explotar oro y cobre en un área cercana a la Laguna del Diamante, importante reservorio hídrico de la zona. Ese mismo año se conformó el "Frente Diamante", grupo constituido por diversos actores tales como uniones vecinales, entidades agrícolas, productores agrícola-ganaderos, concejales, profesionales independientes, empresarios del turismo y docentes y bajo el liderazgo inicial del presidente de la Sociedad Rural de San Carlos. Paralelamente, se organizaron los "Vecinos Autoconvocados de San Carlos", de características asamblearias y más proclives a desarrollar acciones directas quienes, pese a diferenciarse del perfil más institucional y legal propuesto por el Frente Diamante, lograron trabajar conjuntamente.

Hacia 2004, en la ciudad de San Rafael, se inició el conflicto minero al conocerse, por un lado, las intenciones de la Comisión Nacional de Energía Atómica (CNEA) de reabrir las explotaciones de uranio de Sierra Pintada ${ }^{15}$

El complejo Sierra Pintada fue operado por la CNEA en San Rafael desde 1979 hasta 1997. La explotación incluyó además una planta de producción de concentrado de uranio y se estima que el valor sin haber remediado el pasivo ambiental anterior y, por otro lado, los permisos de cateo otorgados a la minera Maple. Con el impulso de productores agrarios, sectores viñateros, y organizaciones no gubernamentales, nucleados en torno a una fuerte participación de la Cámara de Comercio, Industria y Agropecuaria de San Rafael, se conformó la "Multisectorial del Sur por un desarrollo sustentable", con un marcado matiz institucional.

En General Alvear se conformó en 2006 la asamblea "Autoconvocados de Punta del Agua" al conocerse las concesiones mineras otorgadas río arriba de la ciudad. Rápidamente, la vinculación de los asambleístas con organizaciones formales, fundamentalmente la Cámara de Comercio, Industria, Agricultura y Ganadería de General Alvear dio lugar a la conformación de la "Multisectorial de General Alvear", nucleando cerca de 40 entidades de la sociedad civil y de índole empresarial, sindical, política y educativa.

La vinculación entre actores asamblearios y sectores formales y vinculados a las dinámicas productivas se dio al unísono de fuertes episodios de acción directa. La conformación de la Asamblea por el Agua Pura (AMPAP) como instancia provincial de articulación, por un lado y los cortes de ruta y movilizaciones crecientes y masivas, por otro lado, presionaron fuertemente al ejecutivo provincial por la prohibición de la megaminería.

En Mendoza se han conseguido avances parciales y permanentes de limitación a la

del mineral remanente es cercano a los 700 millones de dólares. Actualmente el yacimiento de Sierra Pintada forma parte del Proyecto de Restituciyn Ambiental de la Minerнa del Uranio (PRAMU) (CNEA, 2011). 
minería, tales como las cinco ordenanzas locales sancionadas entre 2005 y $2007^{16} \mathrm{O}$ la ley $n^{\circ}$ 7.422/05 de ampliación de la reserva ambiental de la laguna del Diamante que suspendió cateos mineros en el Valle de Uco. Sin embargo, también han acontecido reveses para los intereses de las resistencias cuando, en diciembre de 2006, el por entonces gobernador Cobos derogó, bajo pretexto de inconstitucionalidad, la ley de prohibición minera 7.627/06. Esta decisión no hizo más que multiplicar el rechazo a la minería e incrementar en los meses subsiguientes las acciones de resistencia.

Durante los momentos previos a la sanción de la ley $\mathrm{n}^{\circ} 7.722 / 07$ con un epicentro en General Alvear y apoyo en el Valle de Uco, se mantuvieron cortadas las principales rutas provinciales durante aproximadamente veinte días. En este contexto de cortes y fuertes movilizaciones sociales, el ejecutivo provincial envió, en junio de 2007, un proyecto de ley a los fines de ser considerado y observado por la Multisectorial de General Alvear. Este escenario es emblemático respecto de la combinación entre acciones contenciosas y participación institucional. El mismo tiempo que mantenían cortada las principales rutas mendocinas debatían y corregían el contenido del proyecto de ley. A partir de la letra original, y con el aporte de una comisión designada por la gente de General Alvear, de legisladores provinciales muy cercanos a las resistencias y demás representes de las organizaciones, tomó cuerpo el proyecto que, presentado bajo el nombre del senador Walter Saenz (UCR), se convertiría finalmente en la ley 7.722/07.

San Carlos (1.068/05); San Carlos (1123/06); Tunuyán (1940/06); Tupungato (001/07); General Alvear (3377/07).
La incorporación del instrumento legal y el derecho ambiental a la contienda fue favorecida por la presencia de actores y organizaciones más formales familiarizados con dicho lenguaje y por una progresiva socialización de conocimientos y experiencias entre las organizaciones asamblearias 0 de base.

La experiencia de San Rafael ilustra el primero de los dos elementos señalados. A partir de la disponibilidad de recursos y la contratación de abogados especialistas por parte de la reconocida Bodega Bianchi, la Multisectorial del Sur interpuso, en marzo de 2005, un recurso de amparo mediante el cual se exigió la no reapertura de las explotaciones de uranio de la CNEA. En respuesta a la misma, el Juzgado Federal de San Rafael dictó la medida de "no innovar". Luego de un proceso judicial en el cual el organismo estatal apeló el fallo, finalmente, en febrero de 2009, la Cámara Federal de Apelaciones Provincial confirmó la medida cautelar de no innovar, en la cual se ordenaba a la CNEA la abstención del inicio de la explotación hasta no remediar la totalidad de los pasivos ambientales generados en las anteriores fases productivas (Larregle 2010).

La "Multisectorial del Sur", en cuadernillos informativos repartidos durante el conflicto, estructuró su argumentación pública mediante una cuidada y precisa apelación al derecho ambiental:

NOS OPONEMOS ADEMÁS A LA EXPLOTACIÓN DE LA MINA DE URANIO PORQUE SABEMOS QUE: (Mayúsculas en el original)

1- Tenemos derecho a elegir nuestra calidad de vida.

8- Porque el Artículo 41 de la Constitución de la Nación Argentina nos dice: "Todos los habitantes gozan del derecho a un ambiente sano, equilibrado, apto para el desarrollo humano y para que las actividades productivas 
satisfagan las necesidades presentes sin comprometer las de las generaciones futuras; y tienen el deber de preservarlo. El daño ambiental generará prioritariamente la obligación de recomponer, según establezca la ley".

9- Porque gracias a la Ley general del Ambiente $\mathrm{N}^{\circ} 25.675$ podemos exigir una gestión sustentable y adecuada al ambiente, la preservación y la protección de la diversidad biológica y la implementación del desarrollo sustentable. Esta ley promueve el mejoramiento de la calidad de vida tanto de las generaciones presentes como futuras (Multisectorial del Sur, s/d)

Por su parte, al interior de las asambleas ambientales, y a pesar de su predilección por la acción directa, fue creciendo progresivamente tanto la valoración del instrumento legal como la apropiación del derecho ambiental:

\begin{abstract}
"Y empezamos a ver y a estudiar leyes, bueno busquemos, no podemos estar todo el tiempo haciendo el corte informativo o marchas. Fue un proceso largo entender la parte legal de todo esto (...) pero hubo mucho asesoramiento de compañeros que trabajando en Recursos Naturales de la provincia se fueron acercando a la lucha y también algunos abogados. En Mendoza tuvimos la suerte de que ya estaba la ley de La Rioja y otras similares, entonces tomamos esas leyes y tratamos de mejorarlas. Acá en Mendoza prohibimos el uso de cianuro, mercurio, ácidos y de cualquier otra sustancia tóxica y le agregamos "en cualquier etapa, exploración, cateo, explotación" (Asambleísta San Carlos, La Consulta, Entrevista, Junio 2014).
\end{abstract}

"El que está muy comprometido con el caso comienza a buscar maneras de argumentar sus ideas. $Y$ aquí han venido mujeres ancianas que empiezan a encontrarse con leyes que decían que tenían derecho al ambiente sano, que con errores conceptuales y eso, demostraban que se estaban apropiando del derecho, que ahí encontraban una herramienta para poder operar. Lo que había que hacer era ajustar conceptos" (Presidente ONG Oikos Ambiental, Ciudad de Mendoza, Entrevista, Junio 2014).

\subsection{El caso de Córdoba}

Laexperienciacordobesa puede esquematizarse a partir de tres elementos centrales: una fuerte red de asambleas de distintas localidades articuladas entre sí; la sanción de sucesivas normativas locales que prohibieron la minería en territorios municipales $\mathrm{y}$; un significativo y protagónico rol de las resistencias en la redacción y la discusión legislativa de la ley 9.526/07.

Las resistencias tuvieron origen, hacia principios de 2007, a partir del conocimiento de prospecciones mineras por parte Red Mineral Corporation en el Valle de Ongamira ${ }^{17}$. Ante esta situación, se conformó la asamblea ¡Ongamira Despierta! con el objetivo de frenar la minería a cielo abierto. Mediante un fuerte trabajo de difusión y socialización de experiencias, en apenas poco más de un año se conformaron más de 25 asambleas distribuidas por gran parte del territorio cordobés. Esta experiencia combino una efectiva acción en el territorio local con una articulación regional en ¡Ongamira Despierta! que fortaleció la resistencia a nivel provincial. Con una lógica de acción similar, hacia 2008 se conformó ¡Traslasierra Despierta!, la cual nucleó distintas asambleas de las localidades del valle homónimo ${ }^{18}$.

La sanción de distintas ordenanzas locales de restricción minera constituyó un exitoso proceso escalar, entendido como una alteración ascendente en el nivel de las arenas comprometidas en la acción colectiva (Tilly, 2001). El conocimiento de las experiencias de otras latitudes, como Esquel, Río Negro y Mendoza, sumado a un expertise técnico y legal creciente, favoreció una valoración positiva de las ordenanzas municipales. De

\footnotetext{
17 ngamira es un valle ubicado al norte de las Sierras Chicas, ubicado a encuentra a $110 \mathrm{~km}$ de la ciudad de Córdoba.

18 El valle de Traslasierra se ubica al oeste de la provincia de Córdoba y sus ciudades más importantes son Mina Clavero y Villa Dolores.
} 
tal modo, entre septiembre de 2007 y abril del 2008 se consiguieron más de 15 normativas de prohibición de la minería metalífera y nuclear en el nivel local ${ }^{19}$.

En mayo de 2008, los miembros de ¡Ongamira Despierta, con el apoyo de ¡Traslasierra Despierta! y otras organizaciones, entregaron en el Ministerio de Gobierno, en la Secretaría de Minería y en la Legislatura Provincial un Proyecto de Ley acompañado de 80.000 firmas. En este caso es emblemático que los mismos contendientes que impulsaron la protesta y la conformación de asambleas de base fueran los encargados de redactar la letra del proyecto. La iniciativa, de redacción conjunta y colectiva a partir de la colaboración de abogados y expertos, contaba con 6 artículos y centraba su objetivo en la prohibición de la actividad minera metalífera y nuclear a cielo abierto en toda la provincia de Córdoba.

Días después, Juan Schiaretti, el por entonces gobernador, envió el Proyecto de Ley de prohibición de actividades metalíferas y minerales nucleares a la legislatura, siendo de tal forma el Poder Ejecutivo el iniciador de la normativa socialmente exigida. El proyecto de prohibición de minería metalífera a cielo abierto y de minerales nucleares rápidamente fue tratado en comisiones para su posterior votación en el recinto. Finalmente el 24 de septiembre de 2008, con un significativo número de contendientes

19 Villa del Totoral (Ordenanza 20/07), Capilla del Monte (Ordenanza 2020/07), San Marcos Sierras (Ordenanza 505/07), La Cumbre (Ordenanza 41/07), Villa Giardino (Ordenanza 706/07), La Falda (Ordenanza 2105/07), Huerta Grande (Ordenanza 861/07), Sinsacate (Ordenanza 471/07), Cruz del Eje (Ordenanza 1774/07), Bialet Massé (Ordenanza 907/07), San Esteban (Ordenanza 472/07) Casa Grande (Resolución 41/07), Charbonier (Resolución 10/07), Comunidad Regional de Totoral (Resolución 11/08), Mina Clavero (Resolución 258/07). dentro y en los alrededores de la legislatura, se aprobó con fuerza de ley, sin votos en contra y solamente con dos abstenciones.

La utilización del elemento legal al repertorio de lucha de las resistencias cordobesas fue parte importante de su proceso contencioso y del éxito obtenido en limitar la megaminería. La experiencia cordobesa contó desde los inicios mismos con una centralidad fuerte del uso del derecho ambiental. Esta particularidad fue impulsada tanto por la socialización de experiencias previas como por la presencia de la abogada ambientalista Marina Martínez Meijide, quien fuera parte de las reuniones fundacionales de ¡Ongamira Despierta! y nexo clave con los actores institucionales.

Adicionalmente, es destacable que la provincia cuenta con avanzados marcos legales ambientales que han sido objeto de la argumentación normativa de las asambleas. La constitución cordobesa sostiene que el estado provincial resguarda el equilibrio ecológico, protege el medio ambiente y preserva los recursos naturales (CP artículo 11) y, garantiza el derecho a gozar de un medio ambiente físico y social libre de factores nocivos para la salud (CP artículo 66). A su vez la Ley de Principios Rectores para la preservación, conservación, defensa y mejoramiento del ambiente $\left(\mathrm{n}^{\circ}\right.$ 7.343/85) declara de interés provincial a los ambientes urbanos, agropecuarios y naturales que mantengan la organización ecológica más conveniente para el desarrollo de la cultura, la ciencia, la tecnología, el bienestar de la comunidad y la permanencia de la especie humana sobre la tierra.

En la experiencia cordobesa se contempló desde los inicios mismos la posibilidad de 
alcanzar por la vía legal una limitación de explotación minera al tiempo que se socializaba al interior de los grupos asamblearios nociones básicas de derecho ambiental:

\begin{abstract}
"Esto de la Unión de Asambleas Ciudadanas fue importante, fue habernos contactado, con un montón de gente que nos decía o empezaban a hablar de las leyes de los lugares que había, entonces decíamos bueno, el hecho de sacar una ley en la provincia de Córdoba es lo que nos va ayudar, (...) Eso fue lo importante, porque nosotros también nos relacionamos con el fiscal Gustavo Gómez y con Enrique Viale ${ }^{20}$, que son abogados ambientalistas. Entonces ellos también te van tirando un conocimiento que nosotros no tenemos. Imagínate, nosotros dos, campesinos acá, hacemos la huerta, qué miércoles podemos hablar de lo jurídico, de la ley y de esas cosas" (Asambleísta ¡Ongamira Despierta!, Villa Giardino, Entrevista, septiembre 2011).
\end{abstract}

Dentro de sus estrategias, ¡Ongamira Despierta! envió una serie de cartas abiertas a actores institucionales, tales como la Presidente de la Nación, el gobernador de Córdoba y secretarías provinciales interesadas en el conflicto. En los textos remitidos a los representantes se aprecia un lenguaje legal que apela tanto a los cuerpos normativos vigentes como a nociones propias del derecho ambiental:

"Conforme lo dicta la Constitución Nacional, la Ley Nacional 25.675 y normas concordantes en la materia, estas zonas debieran ser protegidas de todo emprendimiento minero que afecte o pudiera llegar a afectar el patrimonio natural y cultural que atesoran, todo ello en virtud del principio de equidad intergeneracional y del principio de precaución" (Ongamira Despierta, 2008)...

"Nosotros, como ciudadanos autoconvocados en defensa del agua y de nuestros BIENES naturales y culturales, entendemos que nuestra LUCHA ES TAMBIEN UNA LUCHA POR LOS DERECHOS HUMANOS NO SOLO DEL PRESENTE SINO TAMBIEN DEL FUTURO

El fiscal Antonio Gustavo Gómez (Fiscal General ante la Cámara Federal de Tucumán) y Enrique Viale (Presidente de la Asociación Argentina de Abogados Ambientalistas) son muy reconocidos por sus trabajos vinculados a derecho ambiental.
(Mayúsculas en el original) (...) Exigimos la prohibición de todo emprendimiento minero metalífero a cielo abierto y/o todo aquel que emplee técnicas de lixiviación con sustancias tóxicas en sus procesos de prospección, exploración, extracción y explotación y/o industrialización de minerales a desarrollarse en todo el territorio nacional (...) El cumplimiento de la Constitución Nacional en su Art. 41 (...) y la derogación de la Ley Nacional 24.169 de Inversiones Mineras, Ley 25.161 y normas concordantes" (Ongamira Despierta, 2008).

Junto al proyecto de ley entregado al ejecutivo, ¡Ongamira Despierta! presentó los fundamentos de la ley. El documento, de redacción conjunta y colectiva, construyó su argumentación central en base al deber gubernamental de cumplir con las nociones básicas del derecho ambiental y marcos legales internacionales, nacionales y provinciales en materia ambiental ${ }^{21}$. La calidad técnica y la precisión legal de dichos fundamentos han sido destacadas por distintos legisladores durante el trámite legislativo. En una similar apreciación Marcelo Falo, mano derecha del gobernador, jefe de bancada oficialista y quien fuera el encargado de negociar con los asambleístas, destacó durante una entrevista:

\begin{abstract}
"Nosotros nos encontramos con un grupo de una inmensa racionalidad, racionalidad y formación intelectual, o sea, no hablamos con el corazón y con preconceptos y prejuicios, acá se hablaba con conceptos, con formación. Y hay una persona que para mí fue clave, que fue la Dra. Martínez Meijide. Ella como especialista en temas de derecho ambiental tenía mucho peso en ¡Ongamira despierta! Ella es una mujer muy comprometida, súper
\end{abstract}

21 En ellos aparecen citados: El Artículo 41 de la Constitución Nacional; el Principio 6 de la Declaración de Estocolmo de 1972 emanada de la Conferencia de las Naciones Unidas sobre Medio Ambiente; el Principio 8 de la Declaración de Rio de 1992, producto de la Conferencia de Naciones Unidas sobre el Medio Ambiente y Desarrollo, el Pacto Internacional de Derechos Civiles y Políticos; el principio precautorio establecido en la Constitución Nacional, en la ley nacional 25.675 y normas concordantes; la ley provincial 8066 de Bosques y Tierras Forestales; la ley provincial 8936 de uso de suelos; los artículos 4, 11 y 66 de la Constitución Provincial que refieren al derecho humano al ambiente y los antecedentes de leyes provinciales que prohíben la minería en otras jurisdicciones argentinas. 
racional en sus planteos. Ella contribuyó muchísimo, primero, ella confió en mí, y siempre las organizaciones sociales desconfían de los políticos. Ella confió, y nosotros el proyecto lo hicimos con ella, ella lo conoce en la génesis. Fue clave porque le puso mucho condimento técnico, es abogada y ambientalista. Ella se convirtió en una aliada fundamental para la sanción de la ley" Marcelo Falo, Ciudad de Córdoba, Entrevista, julio 2014).

\subsection{Más allá de la incidencia legislativa exitosa.}

La utilización de una estrategia legal y la apropiación de los derechos ambientales han sido factores de gran importancia no solamente para la sanción de las mencionadas regulaciones antimineras sino también para defensa. En los meses posteriores a sus respectivas sanciones tanto la ley de Mendoza como la de Córdoba fueron objeto de diversos pedidos de inconstitucionalidad por parte de actores ligados a cámaras mineras y la actividad nuclear.

En Córdoba, durante los años en los cuales la norma se mantuvo judicializada, se han realizado campañas puntuales en defensa de la constitucionalidad de la ley, con recolección de firmas y jornadas informativas en un clima de relativa latencia del conflicto ambiental. Finalmente, en agosto de 2015 el Tribunal Superior de Justicia de Córdoba ratificó la plena constitucionalidad de la ley $n^{\circ}$ 9.526/08 y desestimó la acción declarativa de inconstitucionalidad que habían planteado los representantes de la Cámara Empresaria Minera de la Provincia de Córdoba (Cemincor) y de la Asociación de Profesionales de la Comisión Nacional de Energía Atómica y la Actividad Nuclear (APCNEAN). El máximo tribunal manifestó que la ley constituye una norma minera complementaria a las nacionales y que la misma fue dictada dentro de las competencias propias de la provincia en materia ambiental (La Voz del interior 2015).

En el caso de Mendoza las presiones por derogar la ley 7.722 y avanzar con la minería a cielo abierto han sido mayores. Partiendo de doce pedidos de inconstitucionalidad ${ }^{22}$ de la norma, desde presentaciones de proyectos legislativos complementarios hasta intentos de explotación minera con métodos extractivos alternativos ${ }^{23}$ han buscado explotar el elevado potencial minero provincial ${ }^{24}$.

La utilización de un lenguaje de derecho, en combinación con una sostenida acción directa, ha sido central en la defensa de la constitucionalidad de la ley 7722 . Una muestra de esto se aprecia en la precisa argumentación legal que construyera la Asamblea Mendocina por el Agua Pura Frente en un comunicado de prensa frente a un intento legislativo de limitar la aplicación de la ley a dos artículos concretos del código minero 25 :

22 Los pedidos de inconstitucionalidad fueron presentados por: Asociación de Profesionales de la Comisión Nacional de Energía Atómica y la Actividad Nuclear; Minera del Oeste SRL y Desarrollo de Prospectos Mineros; Minera Agaucu SA; Desarrollo de Prospectos Mineros Argentinos SA; Raúl Concina por sí y por Minera Agaucu SA; CognitoLimited, dos por Minera Ríos de la Plata y Minera San Jorge.

23 El proyecto cuprífero de San Jorge fue el caso más significativo. En un intento técnico por esquivar la prohibición del uso de la lixiviación en la industria minera, la compañía Coro Mining propuso el método de flotación para la explotación del yacimiento. La intervención de distintos actores de las resistencias y una fuerte presión social precipitó el rechazo legislativo a la Declaración de Impacto Ambiental (DIA) del proyecto (Según el artículo 3 de la ley 7.722 la DIA de proyectos mineros metalíferos debe ser ratificada por ley).

24 El sector minero mendocino estima que el valor bruto del mineral contenido in situ de los 20 proyectos más relevantes es cercano a los 350 mil millones de dólares. El desarrollo de este potencial minero demandaría una inversión cercana a los 17 mil millones de dólares y podría aportar cerca de 40.000 empleos (CAMEM 2014).

25 En el 2014 los diputados Muñoz, Guerra y Ramos (FPV-PJ) presentaron un proyecto modificatorio de la ley 7.722 a partir de 
"Ratificamos que la 7722 es constitucional, ya que se ajusta a los principios de la política ambiental establecidos en el artículo $4^{\circ}$ de la Ley Nacional 25675, que es una ley de presupuestos mínimos, según el artículo 41 de la Constitución Nacional. Por el contrario, incluir parámetros del Código de Minería de la Nación dentro de una ley ambiental sí es inconstitucional. Es decir que limitar la aplicabilidad de la Ley 7722 a los artículos 33 y 36 del Código de Minería contradice a la Constitución Nacional, a la Ley Nacional 25675 ("Ley General del Ambiente") y atenta contra el derecho a un ambiente sano, garantizado por el artículo 41 de la Constitución" (AMPAP 2014).

En diciembre de 2015, y luego de varias controversias y un proceso judicial dilatado por casi 7 años, la Suprema Corte de Mendoza determinó, en fallo unánime de sus siete jueces, la constitucionalidad de la ley $7722 / 07$. La experiencia mendocina es altamente relevante para ilustrar la combinación entre contienda y participación política institucional. Durante el derrotero del conflicto, los mismos integrantes de las asambleas han sabido ocupar lugares estratégicos dentro del Consejo Provincial de Ambiente (Wagner, 2010) y jugar roles claves durante audiencias públicas ambientales ${ }^{26}$ (Segura, 2011).

\section{Conclusiones. Potencialidades y limitaciones en la relación entre derechos ambientales y participación.}

El desembarco de la minería a cielo abierto y la consagración de los derechos ambientales

los artículos 33 y 36 del Código Minero. La propuesta hubiera significado que la ley sólo mantendría su vigencia a menos de 50 metros de los ríos, a menos de 40 metros de las casas y a menos de 30 metros de acueductos y canales.

26 La más emblemática ha sido la Audiencia Pública del yacimiento San Jorge. En dicha audiencia 143 personas hablaron contra la mina y, con un elevado grado de argumentación técnica y legal, expusieron irregularidades del proyecto. Finalmente, y en el marco de una creciente movilización social, la legislatura mendocina no aprobó el permiso de operación minero. coincidieron en Argentina hacia finales de la última década del siglo veinte. Estos procesos habilitaron una contradicción potencial entre el derecho constitucional a un ambiente sano y un entramado normativo minero hecho a medida de un sector productivo de creciente importancia. El paso de esta contradicción latente a una tensión manifiesta fue fruto de la intervención de las resistencias sociales y su apelación al derecho al ambiente como repertorio de acción contra la minería.

A partir del estudio de los casos de Mendoza y Córdoba, este trabajo ha demostrado que la incorporación de un discurso de derecho a las acciones de resistencia ha sido exitosa y que favoreció la restricción de la minería a cielo abierto en distintas provincias argentinas. La incorporación del instrumento legal a las luchas de las resistencias sociales se vio favorecida por tres elementos: la pluralidad de actores involucrados y la socialización de experiencias; la capacidad de los contendientes de complementar acciones contenciosas con otras canalizadas institucionalmente; y; por último, los resultados relativamente exitosos conseguidos a partir de esta apelación al elemento legal.

En primer lugar, los procesos analizados evidenciaron el accionar de una pluralidad de actores en contra de la minería. Más allá de la centralidad de las asambleas socioambientales como núcleo duro de las resistencias, la riqueza analítica de los casos deriva de las múltiples vinculaciones entre una variedad de actores sociales, económicos y políticos. Este entramado de lenguajes favoreció el intercambio de conocimientos y la incorporación del derecho ambiental a los repertorios colectivos.

En segundo lugar, quienes emprendieron acciones de resistencia lograron combinar, de 
manera estratégica, la participación contenciosa e institucional para conseguir sus objetivos y hacer reales los derechos ambientales. Los contendientes lograron superar miradas esquemáticas que conciben las prácticas contenciosas y la participación institucionalizada como modalidades antagónicas y avanzaron en la consecución de sus metas. Los derechos ambientales en abstracto aparecen como derechos "dormidos" o "adormecidos" y las resistencias los entendieron como derechos a ser conquistados mediante una participación entendida en términos amplios. Los sucesos de Mendoza y Córdoba constituyen experiencias de ampliación de ciudadanía ambiental donde la sanción y defensa de normas de restricción minera vuelven disfrutable el enunciado constitucional del artículo 41.

En tercer lugar, la utilización de una estrategia legal y la apropiación de los derechos ambientales han sido exitosas e impulsaron la sanción de leyes provinciales de prohibición minera en Mendoza y en Córdoba. Al respecto bien vale hacer dos aclaraciones. Por un lado, se identifican también otras experiencias de regulaciones antimineras en el nivel subnacional argentino -como las experiencias de Chubut o Río Negro- donde el discurso de derecho ha sido un factor de relevancia clave. Incluso más, los casos estudiados aquí muestran que el discurso de derecho y el accionar legal también ha sido un elemento de importancia para sostener y mantener los logros normativos frente a las presiones y los pedidos de inconstitucionalidad de las leyes de prohibición minera. Por otro lado, es preciso señalar que la incorporación del elemento legal a los repertorios de acción de las resistencias no implica por sí mismo tener éxito en los reclamos. Esto quiere decir que no en todas las provincias argentinas donde se aplicó la estrategia legal se han obtenido resultados efectivos. En provincias como Catamarca, San Juan y Santa Cruz, las resistencias sociales, con distintas características y variados niveles de intensidad, no han podido restringir el avance de la actividad pese contar con la modalidad legal como parte de sus repertorios ${ }^{27}$.

Se reflexiona también sobre una característica particular de la apelación a la estrategia legal en los conflictos entre minería y ambiente, la cual es factible de profundizar en futuros trabajos. Luego del plebiscito de Esquel, y exceptuando el caso de Loncopué en $2012^{28}$, la búsqueda de avanzar hacia el ambiente saludable y de la restricción minera mediante plebiscitos municipales o provinciales fue restringida. En otras palabras, las respectivas decisiones estatales en materia minera lejos estuvieron de incorporar criterios consultivos amplios. De tal forma, y como señala Delamata (2013), hay un avance del paradigma de "leyes sí, plebiscitos no". En tal sentido, la refrenda ciudadana se mostró, parafraseando a Bobbio (1986), como "promesa incumplida de una democracia más participativa post reforma constitucional de 1994. Esto refuerza el rol clave de la participación

27 Ciertos estudios han analizado por qué en ciertas provincias argentinas es posible limitar la megaminería mientras que en otras no. Christel (2016) afirma que las características del sistema político y la matriz económica conforman Estructuras de Oportunidad Subnacional que favorece o dificulta las posibilidades de incidir en las legislaciones mineras provinciales. Por su parte (Schein 2015) sostiene que tales diferencias se explican por las particularidades del régimen político subnacional.

28 En junio de 2012 en Loncopué, provincia de Neuquén, un 82,08\% de los ciudadanos se expresó en contra de los emprendimientos megamineros. Previamente al plebiscito, en 2009, distintas asambleas y comunidades mapuche detuvieron un proyecto cuprífero mediante sentencia judicial a partir de la apelación a la prevalencia del derecho indígena y la obligatoriedad de consulta a los pueblos originarios según el Convenio 169 de la OIT y la Declaración de las Naciones Unidas sobre los Derechos de los Pueblos Indígenas (Delamata 2103). 
en el avance del derecho al ambiente dado que la garantía del ambiente sano no fue facilitada mediante una participación amplia propia de los derechos procedimentales que traen aparejados los derechos ambientales, sino que tuvo que ser conquistada.

Por último, y a la luz de su productividad normativa, todo parece indicar que la apelación al instrumento legal será una estrategia de acción que se seguirá aplicando al son de nuevas problemáticas ambientales. El escenario continúa siendo complejo, los marcosnormativos son objeto de disputa, las leyes se hacen y deshacen en un equilibrio inestable de intereses y pugnas. Los recursos minerales se agotan, pero aún se descubren nuevos yacimientos a merced de las presiones extractivistas. Es difícil saber cómo se resolverán, a futuro, las controversias ambientales. Pese a esto, sí se puede afirmar que mucho se escribirá sobre ellas y el derecho ambiental será un protagonista privilegiado.

\section{Bibliografía}

AMPAP. 2014. Comunicado de prensa de las Asambleas Mendocinas por el Agua Pura. Ciudad de Mendoza. http://www. opsur.org.ar/blog/2014/06/13/mendoza-repudiamos-el-intentode-modificacion-de-la-ley-7722/ (Consultado en enero de 2016).

Azuela, A. 2006. Visionarios y pragmáticos: una aproximación sociológica al derecho ambiental. México DF: UNAM/Fontamara.

Bobbio, N. 1986. El futuro de la democracia. México DF: Fondo de Cultura Económica.

Cámara Argentina de Empresarios Mineros. 2013. Minería argentina. Todas las respuestas. Aspectos Económicos. Buenos Aires: CAEM.

Cámara de Diputados de la Nación, 1993. "Anexo presentado por diputado Gómez Centurión-Partido Bloquista"- Versión Taquigráfica. Sesión 17 de abril.

CAMEM. 2014. "La minería que Mendoza no explota". Ciudad de Mendoza. Disponible en: http://www.miningpress.com.ar/ nota/262554/camem-la-mineria-que-mendoza-no-explota-crisishidrica-. (Consultado en mayo de 2015).

Christel, L.2016. Resistencias sociales y legislaciones mineras en las provincias argentinas: los casos de Mendoza, Córdoba, Catamarca y San Juan (2003-2009). Tesis de Doctorado, Universidad Nacional de San Martin.

Christel, L. y Gutiérrez, R.A. 2017.Making Rights Come Alive: Enviromental Rights and Modes of Participation in Argentina. Journal of Environment \& Development. 26(3) 322-347.

Christel, L. y Torunczyk, D. 2017. Sovereignties in Conflict: Socio-environmental Mobilization and the Glaciers Law in Argentina. European Review of Latin American and Caribbean Studies (ERLACS). 104,47-68.

CNEA. 2011. "Plan Estratégico. 2010-2019". Buenos Aires: Ministerio de Planificación Federal. Buenos Aires, Argentina. Disponible en: http://www.cnea.gov.ar/Publicaciones-Planestrategico. (Consultado en Julio 2015).
Código de Minería. 1886. Ley №1919.

Constitución de la Nación Argentina. 1994. Santa Fe- Paraná

Collier, D. 2011. Understanding Process Tracing. PS: Political Science and Politics, Vol. 44(4): 823-830

Delamata, G. 2009. "Las resistencias sociales contra la minería transnacional en Argentina. Una aproximación a la escala provincial en la constitución de nuevas identidades políticas”. Em IPSAXXI Congreso Mundial de Ciencia Política, Santiago de Chile, Chile.

Delamata, G. 2013. "Actualizando el derecho al ambiente. Movilización social, activismo legal y derecho constitucional al ambiente de "sustentabilidad fuerte" en el sector extractivista megaminero". Entramados y Perspectivas, 3, 55-90.

Epp, C. 2008. Implementing the rights revolution: Repeat players and the interpretation of diffuse legal messages. Law and Contemporary Problems, 71(4), 41-52.

Gargarella, R. 2010. "El nuevo constitucionalismo latinoamericano", Crítica y Emancipación, 3: 171-188.

Gellers, J. 2015. Environmental constitutionalism in South Asia: Analyzing the experiencesof Nepal and Sri Lanka. Transnational EnvironmentalLaw, 4(2), 395-423.

Gutiérrez, R. A. 2016. "Teoría y praxis de los derechos ambientales en Argentina". Temas y Debates, 30: 13-36.

Hajer, M. 2000. The Politics of Enviromental Discourse: Ecological Modernization and the Policy Process, Oxford: Oxford University Press.

Hannigan, J. 2006. Environmental sociology. New York, NY: Routledge.

Hiskes, R. 2009. The Human Right to a Green Future. Cambridge: Cambridge University Press.

Jeffords, C., y Minkler, L. 2016. Do constitutions matter? The effects of constitutional environmental rights provisions on environmental outcomes. KYKLOS, 69, 294-335. 
Kriesi, H. 1996. La estructura organizacional de los nuevos movimientos sociales en su contexto político. En McAdam, D, McCarthy, J y Zald, D, Movimientos sociales: Perspectivas comparadas. Oportunidades políticas, estructuras de movilización y marcos interpretativos culturales, Madrid: Istmo.

La voz del interior. 2015. "El TSJ ratificó la prohibición de la minería a cielo abierto en Córdoba". 11 de agosto. Disponible en: http://www.lavoz.com.ar/ciudadanos/eltsj-ratifico-la-prohibicion-de-la-mineria-cielo-abierto-encordoba(Consultadoenagosto 2015).

Larregle, D. 2010. "La Corte frenó la reapertura de Sierra Pintada". Diario Los Andes, 17 de diciembre. Disponible en: http://archivo.losandes.com.ar/notas /2010/12/17/corte-frenoreapertura-sierra-pintada-540047.asp. (Consultado en junio 2015).

Ley $\mathrm{N}^{\circ}$ 25.675. 2002. Ley General de Ambiente.

Magnussen, A., y Banasiak, A. 2013. Juridification: Disrupting the relationship between law and politics? European Law Journal, 19(3), 325-339.

Marin, M. 2009. "El No de Esquel como acontecimiento: otro mundo posible". En Svampa, M y Antonelli, M, Minería trasnacional, narrativas del desarrollo y resistencias sociales, Buenos Aires: Editorial Biblos.

May, J. 2013. Constitutional directions in procedural environmental rights. Journal of Environmental Law \& Litigation, 28, 27-51.

Moody, R. 2007. Rocks and Hard Places: The Globalization of Mining. New York: Zed Books.

Multisectorial del Sur. n.d. "El ambiente adecuado para la vida ES un derecho de todos". Disponible en:https:// dl.dropboxusercontent.com/u/25333702/gacetilla \% 20 Multisectorial.pdf (Consultado en enero 2017).

Nogueira Alcalá, H. 2007. El recurso de protección en el contexto del amparo de los derechos fundamentales latinoamericano e interamericano. lus et Praxis. 13, 75-134.

Ongamira Despierta! 2008. "Fundamentos proyecto ley". Disponible en: http://recuperarlonuestro.blogspot. com.ar/2008/05/ongamira-despierta-proyecto-de-ley.html. (Consultado en julio 2015).

Plan Nacional Minero. 2004. Secretaría de Minería. Ministerio de Planificación Federal, Inversión Pública y Servicios. Disponible en: www.infoleg.gob.ar/basehome/actos_gobierno/ actosdegobierno11-5-2009-1.htm (Consultado en marzo 2019)

Robledo, F. 2010. Las garantías judiciales como vías de tutela de los derechos fundamentales en estados de emergencia (in) constitucional. Estudios Constitucionales. 8, 247-292.

Sabsay, D. y Di Paola, M.E. 2008. "Coordinación y armonización de las normas ambientales en la República Argentina". Revista de Derecho de Daños, 3:137-162.

Schein, D. 2015. "Souverainetés en conflit: industrie minière transnationale, politiques ubnationale et mouvements socioenvironnementaux en Argentine: une analyse comparée dans les provinces de Chubut et de Santa Cruz". Tesis de Doctorado en Sociologia, Universite du Quebec a Montreal.

Secretaría de minería de la nación. s/d. La minería en números. Buenos Aires, Argentina. Disponible en: http://www.mineria.gob. ar/pdf/mineriaennumeros.pdf. (Consultado en mayo 2015).

Segura, E. 2010. "Si no lo entendés te lo explico con un dibujito". Disponible en:http://www.mdzol.com/nota/252650 (Consultado en enero 2017).

Shelton, D. 2013. Human Rights and the Environment. Substantive Rights (Legal StudiesResearch Paper 2013-33). Washington, DC: The George Washington University LawSchool.

Snyder, R. 2001. "Scaling Down: The Subnational Comparative Method". Studies in Comparative International Development, 36:93-110.

Svampa, M. y Antonelli, M. Eds. 2009. Minería trasnacional, narrativas del desarrollo y resistencias sociales. Buenos Aires: Editorial Biblos.

Svampa, M.,Bottaro, L. y Sola Alvarez, M. 2009. "La problemática de la minería a cielo abierto". EnSvampa, M. y Antonelli, M, Minería trasnacional, narrativas del desarrollo y resistencias sociales, Buenos Aires: Editorial Biblos.

Tarrow, S. 2011. Power in Movement. Social Movements and Contentious Politics (third Edition). New York: Cambridge University Press.

Tilly, C. 2001. "Mechanisms in Political Processes". Annual Review of Political Science, 4:21-41.

Unión de Asambleas Ciudadanas. 2011. "La Unión de Asambleas Ciudadanas. Construyendo caminos colectivos en defensa de nuestros territorios". Argentina. Disponible en: http:// asambleasciudadanas.org.ar/wp-content/uploads/2012/09/ CuadernilloUAC-para-imprimir.pdf. (Consultado en julio 2015).

Wagner, L. 2010. "Problemas ambientales y conflicto social en argentina. Movimientos socioambientales en Mendoza. La defensa del agua y el rechazo a la megaminería en los inicios del siglo XXI". Tesis de Doctorado en Ciencias Sociales. Universidad Nacional de Quilmes. 
